\title{
A NEW FINITE-SOURCE QUEUEING MODEL FOR MOBILE CELLULAR NETWORKS APPLYING SPECTRUM RENTING
}

\author{
TIEN V. DO* \\ Department of Networked Systems and Services \\ Budapest University of Technology and Economics \\ Magyar tudósok körútja 2, Budapest, Hungary \\ do@hit.bme.hu \\ PATRICK WÜCHNER \\ Faculty of Informatics and Mathematics \\ University of Passau \\ Innstraße 43, 94032 Passau, Germany \\ patrick.wuechner@uni-passau.de \\ TAMÁS BÉRCZES \\ Faculty of Informatics \\ University of Debrecen \\ Egyetem tér 1, P.O. Box 12, 4010 Debrecen, Hungary \\ berczes.tamas@inf.unideb.hu \\ JÁNOS SZTRIK \\ Faculty of Informatics \\ University of Debrecen \\ Egyetem tér 1, P.O. Box 12, 4010 Debrecen, Hungary \\ sztrik.janos@inf.unideb.hu \\ HERMANN DE MEER \\ Faculty of Informatics and Mathematics \\ University of Passau \\ Innstraße 43, 94032 Passau, Germany \\ hermann.demeer@uni-passau.de \\ Received 30 September 2013 \\ Revised 10 April 2013 \& 6 June 2013 \\ Accepted 8 June 2013 \\ Published
}

This paper proposes a retrial queueing model with the finite number of sources to evaluate the performance of spectrum renting in mobile cellular networks. The model incorporates necessary ingredients such as the finite number of subscribers, their impatience and a queue for the outbound service. To consider the specific feature of spectrum renting and the current mobile cellular technology, a variable number of servers that are switched on and off in groups is introduced. We present a novel way to take into account the renting

* Corresponding author. 


\title{
T. V. Do et al.
}

\begin{abstract}
fee, which can be used to fine-tune the operation of the spectrum renting procedure. Numerical results show that it is still profitable to initiate a spectrum renting request at high loads, even if no discount is offered by the frequency bands' owners.

Keywords: Mobile cellular networks; retrial queues; finite-source; impatience; spectrum renting; discount factor.
\end{abstract}

\section{Introduction}

The theory of retrial queues has been applied to model and evaluate the resource contention problem and the distinct user behavior in mobile cellular networks and telecommunication systems (Ajmone Marsan et al., 2001; Almási et al., 2005; Artalejo, 1998, 1999; Artalejo et al., 2007; Artalejo and Gómez-Corral, 2008; Artalejo, 2010; Artalejo and Lopez-Herrero, 2010; Economou and Lopez-Herrero, 2009; Falin and Templeton, 1997; Falin, 1990; Perel and Yechiali, 2010; Do, 2010b, 2011; Tran-Gia and Mandjes, 1997).

A typical classification of works on retrial queues and applications can be based on the assumption about the number of traffic sources. Infinite-source models are treated, e.g., by Ajmone Marsan et al. (2001), Artalejo (2010), Do (2010a), Do (2010b), Do et al. (2013), and Tran-Gia and Mandjes (1997). Finite-source retrial queues are discussed, e.g., by Artalejo and Lopez-Herrero (2012), Almási et al. (2005), Gharbi and Charabi (2012), Kulkarni and Liang (1997), Wüchner et al. (2009), Zhang and Wang (2012), Gharbi and Dutheillet (2011), Yang and Templeton (1987), Wang et al. (2011) and Zhang and Wang (2013). On the one hand, the assumption of the number of infinite sources covers general modeling opportunities and may lead to some efficient algorithms (Ajmone Marsan et al., 2001; Do, 2010a, 2010b; Do and Chakka, 2010; Do et al., 2013; Tran-Gia and Mandjes, 1997) for the performance evaluation of systems. On the other hand, the construction of finitesource models is justified by the inherent fact that the number of subscribers in the specific area of mobile cellular networks is finite.

Spectrum renting is a technique to relieve the temporary capacity shortage of a specific service area in wireless cellular networks, which have been intensively researched by the telecommunication industry in recent years (Buddhikot, 2007; Gandhi et al., 2008; Peha, 2009; Jabbari et al., 2010). Some queueing models for spectrum renting were proposed. Tzeng and Huang (2010) and Tzeng (2009) assumed that user channels can be rented in one unit, which is not realistic. The reason is that the separate blocks of user channels are defined in each frequency band, and each block should be controlled by a single network operator. Do et al. (2012) presented the first queueing model to take into account this technology aspect, but they did not consider the retrial phenomenon in their model. It is worth mentioning that Artalejo et al. (2005) first analyzed a multi-server retrial model with a variable number of active servers, but their results cannot be applied directly for modeling spectrum renting, since servers are switched on and off one-by-one in their model.

Following the common practice applied in the theory of retrial queues for performance evaluation, this paper proposes a novel finite-source retrial queue to model 
spectrum renting in mobile cellular networks. The model incorporates switching servers on and off in groups, considers the finite size of the subscribers' population, their impatience, and has a queue for the subscribers who requested outbound service. Additionally, we present a novel way to take into account the renting fee, which can be used to optimize the spectrum renting procedure. Numerical results are derived and shown that renting an additional frequency band is still desirable in certain circumstances, even if there is no discount from the owners of unused frequency bands.

To our best knowledge, this is the first proposal for the use of the theory of retrial queues to model spectrum renting. Moreover, several model variants could be derived to investigate additional aspects that are not completely explored yet in this paper. Therefore, we hope this article can initiate a new direction for the further exploration of a novel sub-class of retrial queueing models.

The rest of this paper is organized as follows. The review of an applicationspecific technical background that motivates this work is given in Sec. 2. In Sec. 3, the proposed model is presented. Numerical results and their discussion are provided in Sec. 4. Finally, Sec. 5 concludes the paper.

\section{Key Aspects}

This section provides a short overview of some technical aspects we consider in our model. A detailed description of techniques applied for mobile cellular networks can be found in the specifications of the relevant standardization institutes, books, and further literature (Lin and Chlamtac, 2001; Rappaport, 2002).

\subsection{Block of traffic channels}

There are main alternatives for handling multiple access in mobile cellular networks: The Frequency and the Time Division Multiple Access (FDMA, TDMA) techniques (Lin and Chlamtac, 2001) and the Code Division Multiple Access (CDMA). The latter is based on spread-spectrum technology and a code division scheme (Rappaport, 2002). In these alternatives, each frequency band hosts a block of several channels.

To provide service for customers, a specific mobile network operator should (i) purchase the exclusive right for the use of a number of frequency bands in a country, (ii) divide the whole coverage area into a number of regular shaped cells, and (iii) assign a number of frequency bands to each cell. As a result, there will be a number of channels that can be allocated to subscribers in each cell.

\subsection{Spectrum renting}

Deciding on the optimum number of frequency bands to be purchased and assigned to a cell is a non-trivial task. In particular, over-provisioning is costly and underprovisioning might quickly annoy customers due to insufficient service quality. Spectrum renting can be used to relieve temporary shortages in the capacity of mobile 


\section{T. V. Do et al.}

cellular networks and hence provides a flexibility in the management of network resources.

Several alternatives to realize spectrum-sharing models and a spectrum policy reform are suggested by Peha (2009). Based on these, Do et al. (2012) argued that network operators may cooperate with each other in a dynamic market to perform spectrum renting based on some economic principles and a bid mechanism. That is, the owners of spectrum collaborate to increase the efficiency of the spectrum usage and to relieve the temporary capacity shortage of a particular cell in a mobile cellular network. For example, when the number of calls increases in a specific area, a network operator could rent an additional frequency band from another operator to reduce the blocking probability of calls.

\subsection{Outbound or callback service}

Even if spectrum renting is performed, all channels might be busy during peak hours. When the initiation of a call is unsuccessful, the calling subscriber is informed through a signaling channel (e.g., operators send a voice announcement) that by pressing a certain button he or she can request the outbound service. Later, when a free channel is available, operators initiate a call for subscribers that previously requested the outbound service in the first-come-first-served discipline.

\subsection{The impatience of subscribers}

Behavioral psychology concerning the use of service offered by mobile cellular networks includes repeated attempts and abandonments. Both phenomena reflect the impatience of subscribers when all channels are occupied.

Following the arrival of a call, if all the available channels are occupied, a call is not be admitted into a network. Later, a subscriber initiates a repeated attempt for the admission of a call.

An abandonment happens when a subscriber's call becomes rejected and the subscriber gets impatient and gives up after a certain time without getting service.

\section{The System Model}

The finite-source retrial queueing model is illustrated in Fig. 1. It comprises of finite number of sources, the orbit of retrying customers, the queue of customers that requested the outbound service, and the service area including a renting mechanism. The details of the model, taking into account the renting mechanism, are presented below.

A particular cell is assumed to contain a finite user population of $K$ subscribers. Each subscriber initiates a call with rate $\lambda$ as long as it is neither waiting for a channel nor being served. The operator allocates $n$ initial channels from its own frequency bands to serve incoming calls. It is assumed that the operator can also rent $m$ additional frequency bands. Each frequency band accommodates $r$ channels. 


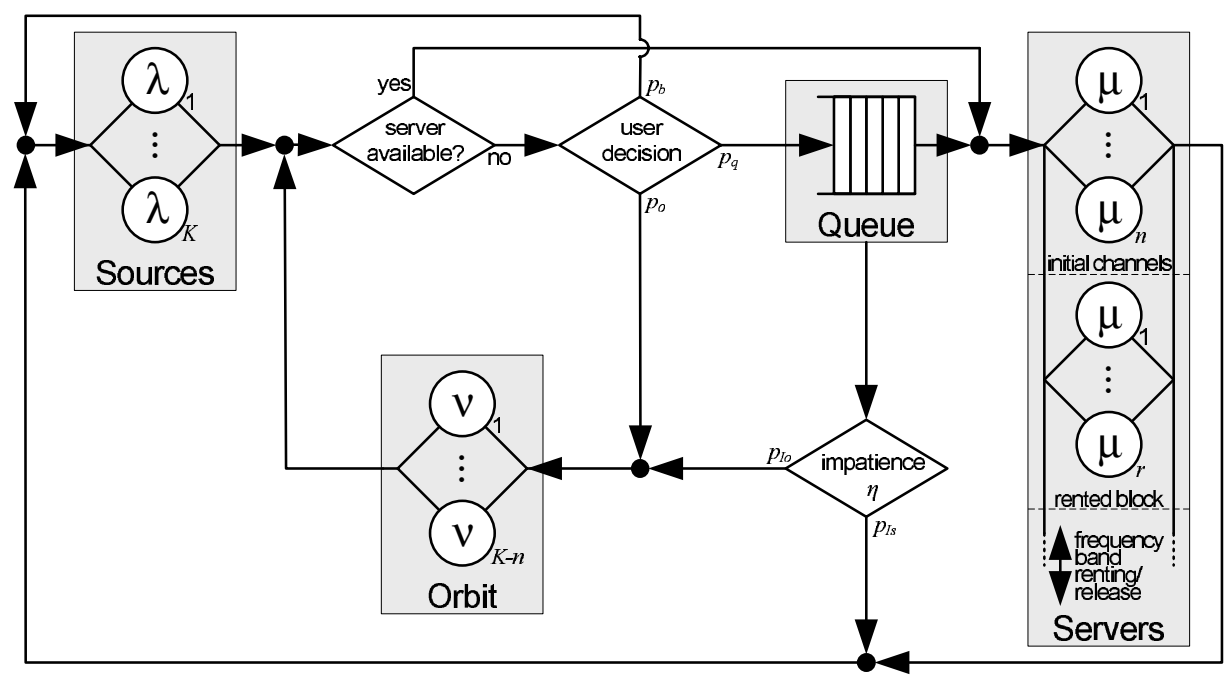

Fig. 1. A retrial queue with components.

Therefore, the maximum number of available channels for calls is $n+m \times r$ if all $m$ frequency bands are rented for the investigated cell.

\subsection{Subscribers' behavior}

The subscribers' behavior, including the outbound service and the impatience of subscribers, is considered in our model as follows. Upon the arrival of either a fresh call from the sources or a retrying call from the orbit, it checks whether there is an idle channel.

(1) If there is an idle channel, the call occupies this channel immediately for a call duration that is exponentially distributed with parameter $\mu$. After the call duration, the channel is released and the subscriber returns to the sources.

(2) If all channels are busy, the subscriber chooses one of the three alternatives:

(i) The subscriber requests the outbound service with probability $p_{q}$. In our model, those subscribers join the virtual "outbound queue". Whenever an idle channel gets available (i.e., a busy channel gets idle or more channels are rented), the system automatically and immediately initiates the call for subscribers "waiting" in the outbound queue following a first-come-firstserved scheduling discipline.

(ii) The subscriber joins the orbit with probability $p_{o}$. Subscribers waiting in the orbit retry to obtain a free channel with retrial rate $\nu$.

(iii) The subscriber gives up and becomes idle, i.e., returns to the pool of sources, with probability $p_{b}=1-p_{o}-p_{q}$. 


\section{T. V. Do et al.}

Note that the subscriber in the outbound queue might get impatient (after an exponentially distributed time) with rate $\eta$. He/she leaves the outbound queue either to the orbit (retrying later) with probability $p_{I o}$ or "goes back" to the pool of the sources (i.e., giving up the call) with probability $p_{I s}=1-p_{I o}$.

\subsection{Spectrum renting}

To ensure the smooth operation related to renting and releasing frequency bands, the operation rule based on a hysteresis control with two thresholds is introduced by Do et al. (2012) as follows:

(1) Initiation of renting a frequency band: Whenever the number of free channels reduces to the lower threshold $t_{1}$ (which is due to the occupation of one channel by a call from either the finite-source pool or the orbit when the number of free channels is $\left.t_{1}+1\right)$, the network operator initiates the request to rent a new frequency band. Let $p_{r}$ denote the probability that the request for a new frequency band is successful.

If the request for a new frequency band fails (with probability $p_{f}=1-p_{r}$ ), the network operator retries with rate $\nu_{r}$ as long as the number of free channels is equal to $t_{1}$ or less.

(2) Release of a frequency band: Whenever the number of free channels increases to $t_{2}+r$, then $r$ channels are returned back to their owner after an exponentially distributed release time with mean $1 / \mu_{r}$. Note that there is a positive release time and the immediate serving of subscribers that requested the outbound queue always has priority over releasing frequency bands.

The two spectrum renting parameters $t_{1}$ and $t_{2}$ are used to tune in the performance of the system. Later, a specific operator returns back rented channels, the higher the chance that more calls will be realized. However, there is a trade-off between the blocking probability and the fees a network needs to pay for a rented spectrum. To make a trade-off, we will define the average profit rate (APR) in Sec. 4.

\subsection{The underlying Markov chain}

To analyze the proposed queueing model (see Fig. 1), we introduce the following notations.

(1) $k(t)$ is the number of active sources at time $t$,

(2) $s(t)$ denotes the number of occupied channels at time $t$,

(3) $b(t)(0 \leq b(t) \leq m)$ is the number of rented frequency bands at time $t$,

(4) $q(t)$ denotes the number of calls in the outbound queue at time $t$,

(5) $o(t)$ is the number of calls in the orbit at time $t$,

(6) $u(t)(u(t) \in\{0,1\})$ indicates whether there is an unsatisfied block rental retrial at time $t$.

Note that $k(t)=K-s(t)-q(t)-o(t)$. 
Table 1. Overview of main model parameters.

\begin{tabular}{llcc}
\hline Parameter & \multicolumn{1}{c}{ Maximum } & Value at time $t$ & Mean $(t \rightarrow \infty)$ \\
\hline Number of active sources & $K$ (population size) & $k(t)$ & $\bar{K}$ \\
Calls in queue & $Q=K-n$ (queue size) & $q(t)$ & $\bar{Q}$ \\
Orbiting calls & $O=K-n$ (orbit size) & $o(t)$ & $\bar{O}$ \\
Busy channels & $n+m \times r$ & $s(t)$ & $\bar{S}$ \\
Number of rented bands & $m$ & $b(t)$ & $\bar{B}$ \\
Activity of block rental retrial & 1 & $u(t)$ & $\bar{U}$ \\
Call generation rate & - & $\lambda$ & - \\
Total call generation rate & $\lambda K$ & $\lambda k(t)$ & $\bar{\lambda}=\lambda \bar{K}$ \\
Impatience rate & - & $\eta$ & - \\
Service rate & - & $\mu$ & - \\
Retrial rate & - & $\nu$ & - \\
\hline
\end{tabular}

The main model parameters are summarized in Table 1 (the complete list of parameter values for the investigation can be found in Table 2). In addition, the system's steady-state probabilities are defined as

$$
p_{s, b, q, o, u}=\lim _{t \rightarrow \infty} P(s(t)=s, b(t)=b, q(t)=q, o(t)=o, u(t)=u) .
$$

To obtain the steady state probabilities and performance measures within the Markovian framework, the mathematical tractability of the proposed model should be preserved. Therefore, we follow the classical approach frequently applied in the theory of retrial queues for the performance evaluation of wireless cellular networks (Ajmone Marsan et al., 2001; Almási et al., 2005; Artalejo, 1998, 1999; Artalejo et al., 2007; Artalejo and Gómez-Corral, 2008; Artalejo, 2010; Artalejo and Lopez-Herrero, 2010; Do, 2010b, 2011; Economou and Lopez-Herrero, 2009; Falin and Templeton, 1997; Falin, 1990; Perel and Yechiali, 2010; Tran-Gia and Mandjes, 1997). That is, all interevent times (i.e., request generation time, impatience time, service time, retrial time, and times related to the spectrum renting) are assumed to be exponentially distributed and regulated by their rate parameter.

Based on the assumptions, the system is modeled by the five-dimensional (5D) Continuous Time Markov Chain (CTMC) $\{s(t), b(t), q(t), o(t), u(t)\}$, which is driven by the following types of events:

(1) the arrival of calls,

(2) the impatience and retrials of calls,

(3) the departure of calls,

(4) the request and retrial process for renting spectrum,

(5) the release of rented spectrum.

Note that in the present case, the unique stationary distribution always exists, because the underlying CTMC is irreducible and the state space of the CTMC is finite. A graphical illustration of the 5D CTMC appears to be infeasible and is omitted in this paper. Suitable analysis tools (like MOSEL-2, see Sec. 4 for details) allow the automatic generation of the CTMC and its infinitesimal generator matrix. 


\section{T. V. Do et al.}

These tools use standard techniques (Takacs, 1962; Bolch et al., 2006) to solve the global-balance equations for the system's steady-state probabilities.

As soon as the steady-state probabilities are known, further interesting performance measures to characterize spectrum renting can be defined as follows:

(1) The blocking probability* is

$$
p_{\text {block }}=\sum_{(s, b, q, o, u) \in X \mid s=n+b r} P(s, b, q, o, u) .
$$

(2) The mean number of rented frequency bands is

$$
\bar{B}=\sum_{u=0}^{1} \sum_{b=1}^{m} \sum_{s=0}^{n+b \times r} \sum_{q=0}^{\min (K-n, K-s)} \sum_{o=0}^{\min (K-n-q, K-s-q)} b P(s, b, q, o, u) .
$$

(3) The mean number of busy channels is

$$
\bar{S}=\sum_{u=0}^{1} \sum_{b=0}^{m} \sum_{s=1}^{n+b \times r} \sum_{q=0}^{\min (K-n, K-s)} \sum_{o=0}^{\min (K-n-q, K-s-q)} s P(s, b, q, o, u) .
$$

(4) The mean queue length is

$$
\bar{Q}=\sum_{u=0}^{1} \sum_{b=0}^{m} \sum_{s=0}^{n+b \times r} \sum_{q=1}^{\min (K-n, K-s)} \sum_{o=0}^{\min (K-n-q, K-s-q)} q P(s, b, q, o, u) .
$$

(5) The mean orbit length is

$$
\bar{O}=\sum_{u=0}^{1} \sum_{b=0}^{m} \sum_{s=0}^{n+b \times r} \sum_{q=0}^{\min (K-n, K-s)} \sum_{o=0}^{\min (K-n-q, K-s-q)} o P(s, b, q, o, u) .
$$

(6) The mean number of active sources is

$$
\bar{K}=K-\bar{S}-\bar{Q}-\bar{O} .
$$

(7) The mean system throughput is

$$
\bar{\lambda}=\bar{K} \lambda
$$

(8) To compute the mean time spent in orbit, we follow the approach by Wüchner et al. (2009). Let $\lambda_{O}$ denote the throughput of the orbit. Using the Little Law, the mean time spent in the orbit (per visit) by every request arriving at the orbit is $\overline{T_{O}^{\nu}}=\frac{\bar{O}}{\lambda_{O}}$. The mean number of visits to the orbit per requests is $e_{O}=\frac{\lambda_{O}}{\bar{\lambda}}$. Hence, the overall mean time spent in the orbit is

$$
\overline{T_{O}}=e_{O} \overline{T_{O}^{\nu}}=\frac{\lambda_{O}}{\bar{\lambda}} \frac{\bar{O}}{\lambda_{O}}=\frac{\bar{O}}{\bar{\lambda}} .
$$

*In this paper, the term blocking probability refers to the probability (as seen by an outside observer) that all initial and rented servers are busy. 
(9) The mean time spent in queue is

$$
\overline{T_{Q}}=\frac{\bar{Q}}{\bar{\lambda}} .
$$

\section{Numerical Results}

In this paper, the MOSEL-2 tool ${ }^{\dagger}$ is applied to compute the steady-state probabilities and the performance measures of the system. In particular, for generating the underlying CTMC and calculating the steady-state probabilities, MOSEL-2 calls SPNP (Ciardo et al., 1989; Hirel et al., 2000), which uses the successive overrelaxation method to solve the system of global-balance equations numerically.

Note that further evaluation tools such as MOSES (Greiner and Bolch, 1995) or TimeNET (German et al., 1995) can be invoked by MOSEL-2. Other evaluation methods, like discrete-event simulation, allows further generalizations for the presented model. For example, the assumption of exponentially distributed state transitions could be relaxed. However, these are out of the scope of this paper.

In this section, we study the scenario where $K=100$ subscribers reside in the investigated cell of a network operator. If not stated otherwise, the values of further parameters are identical to the default model parameter set summarized in Table 2.

Table 2. Numerical values of default model parameters.

\begin{tabular}{llll}
\hline Parameter & Symbol & Value & Unit \\
\hline Normalized traffic intensity & $\rho_{0}=\frac{K \lambda}{n \mu}$ & {$[0.6,4.6]$} & - \\
Number of active sources & $K$ & 100 & $1 / s$ \\
Retrial rate & $\nu$ & 1 & - \\
Prob. that subscriber gives up & $p_{b}$ & 0.1 & - \\
Prob. that subscriber joins the queue & $p_{q}$ & 0.5 & - \\
Prob. that subscriber joins the orbit & $p_{0}$ & $1-p_{b}-p_{q}$ & $1 / s$ \\
Impatience rate & $\eta$ & $1 / 300$ & - \\
Prob. that impatient user goes back to orbit & $p_{I o}$ & 0.8 & - \\
Prob. that impatient user gives up & $p_{I s}$ & $1-p_{I o}$ & - \\
Number of channels per block & $r$ & 8 & $1 / s$ \\
Maximum number of rented blocks & $m$ & 5 & - \\
Number of channels without renting & $n$ & $2 \times r$ & - \\
Service rate & $\mu$ & $1 / 53.22$ & - \\
Block renting threshold & $t_{1}$ & $0<t_{1}<t_{2}<r$ & - \\
Block renting rate & $\lambda_{r}$ & $1 / 5$ & $1 / s$ \\
Block release threshold & $t_{2}$ & $0<t_{1}<t_{2}<r$ & - \\
Block release rate & $\mu_{r}$ & 1 & - \\
Prob. for successfull renting & $p_{r}$ & 0.8 & $1-p_{r}$ \\
Prob. for unsuccessfull renting & $p_{f}$ & $1 / 7$ & \\
Block rental retrial rate & $\nu_{r}$ & & $-s$
\end{tabular}

${ }^{\dagger}$ MOSEL-2 is maintained at the Chair of Computer Networks and Communications, University of Passau, Germany. The project homepage is available at: http://mosel2.net.fim.uni-passau.de/ Accessed on April 8, 2013. 


\section{T. V. Do et al.}

The normalized traffic intensity $\rho_{0}=(K \lambda) /(n \mu)$ is changed in the interval $[0.6,4.6]$ which translates to the blocking probability of approximately $1 \%$ to $2 \%$ for the default model parameter set.

To illustrate the capability of spectrum renting, we first compare a case where spectrum renting is not applied with a case, where the spectrum renting procedure is carried out. In Fig. 2, performance measures versus the normalized traffic intensity are plotted when spectrum renting is not applied. The probability that all $n$ channels are busy reaches 1 when $\rho_{0}=2.1$ for $n=16$. As expected, the performance is degraded by the increase of traffic load. Now, we are going to investigate how spectrum renting can help the network operator in such circumstances.

We compute the performance measures versus the normalized traffic intensity, $t_{1}$ and $t_{2}$. It is observed in Fig. 3 that the change on $t_{1}$ only has a minimal impact on the mean number of rented blocks and on the mean number of busy channels. Figure 4
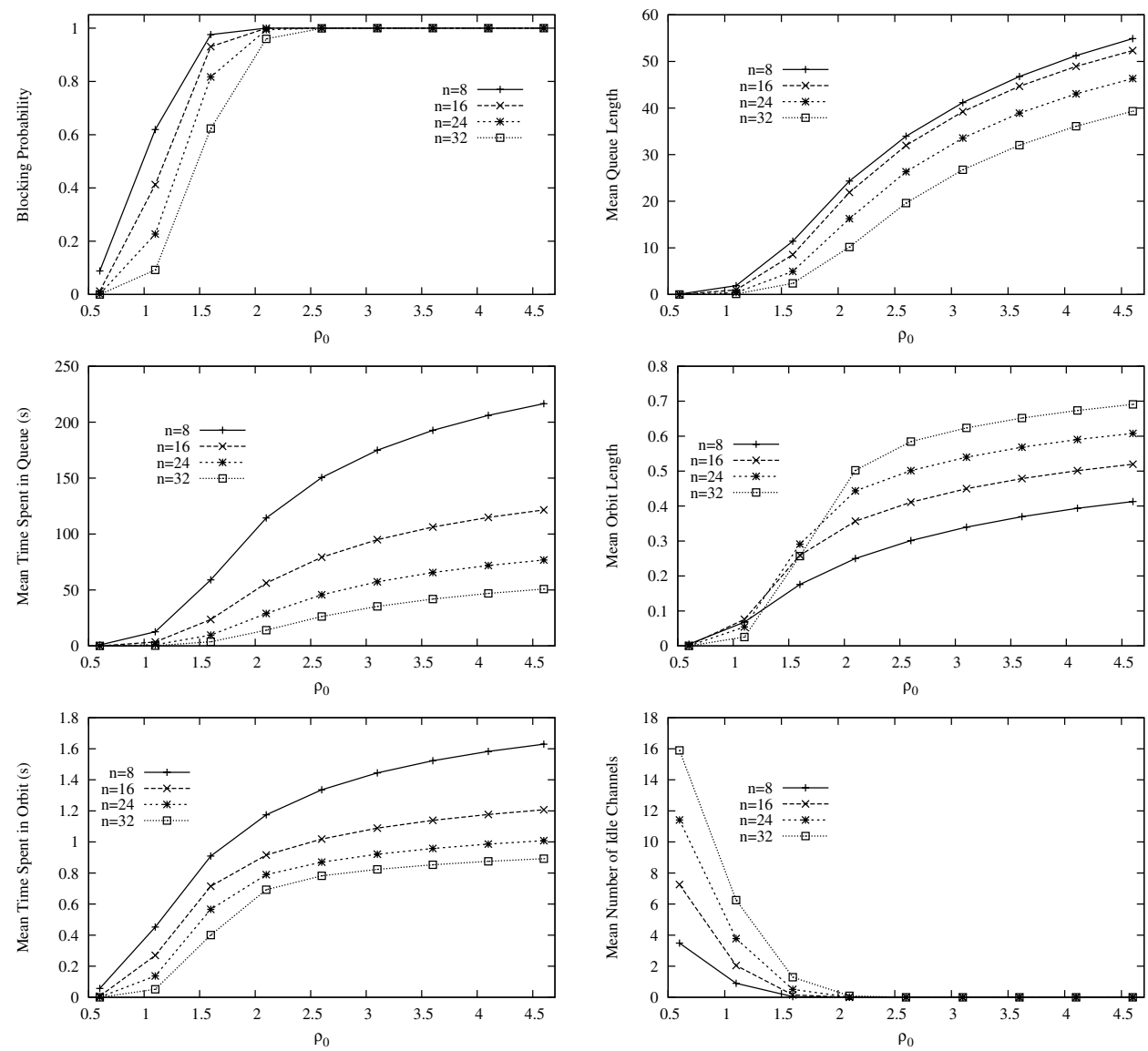

Fig. 2. Performance measures when there is no renting. 

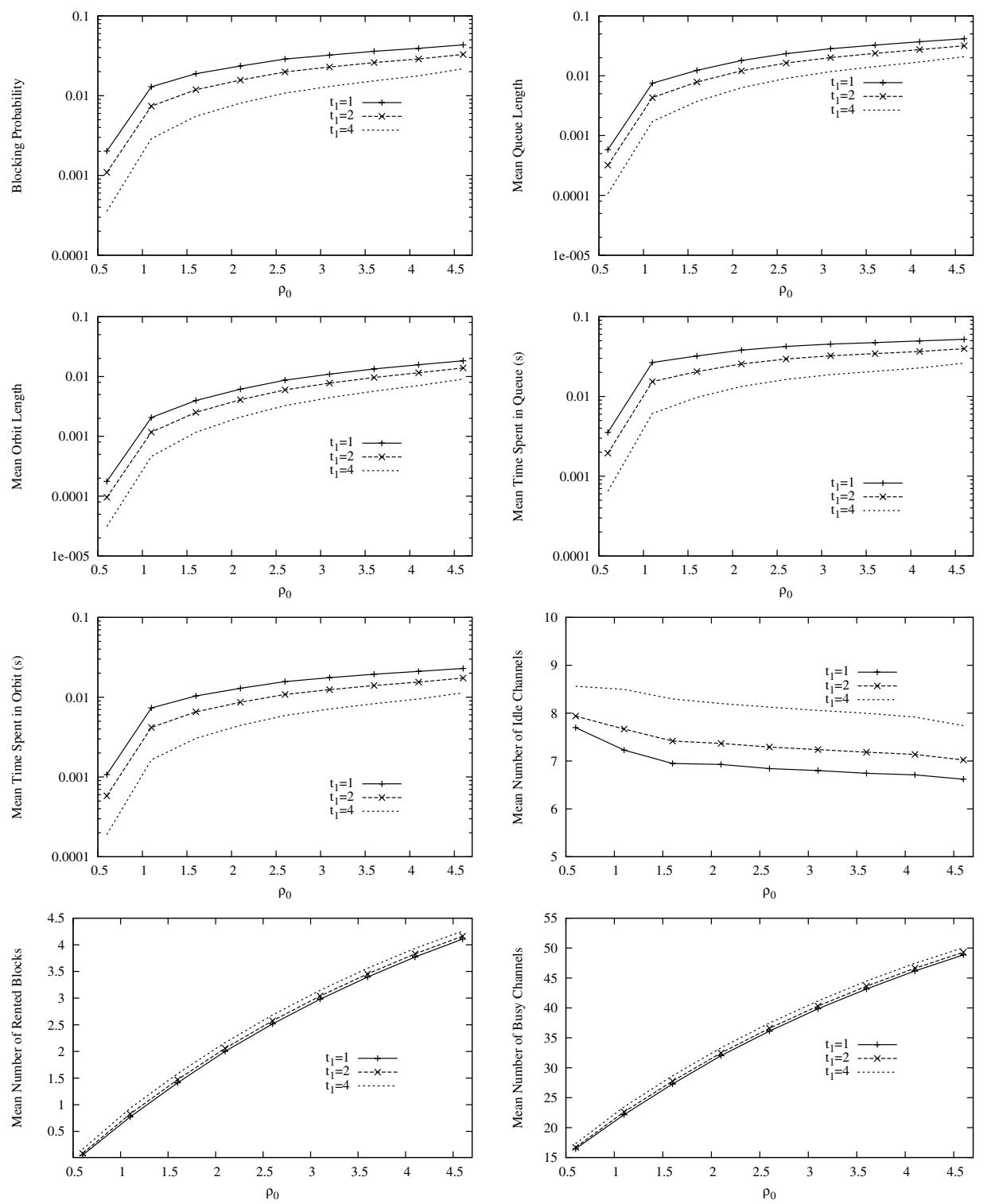

Fig. 3. Performance measures for $t_{2}=6$.

also illustrates that $t_{1}$ has a negligible influence on the probabilities concerning the utilization of rented blocks. Furthermore, Fig. 4 shows that for increasing load, the probability that rented blocks are actually used increases quickly. Hence, the renting of additional blocks is indeed suggested for increasing load.

On the one hand, the higher the value of $t_{1}$ (i.e., the earlier the operator initiates a request for an additional frequency band) is, the better the subscribers' "quality 

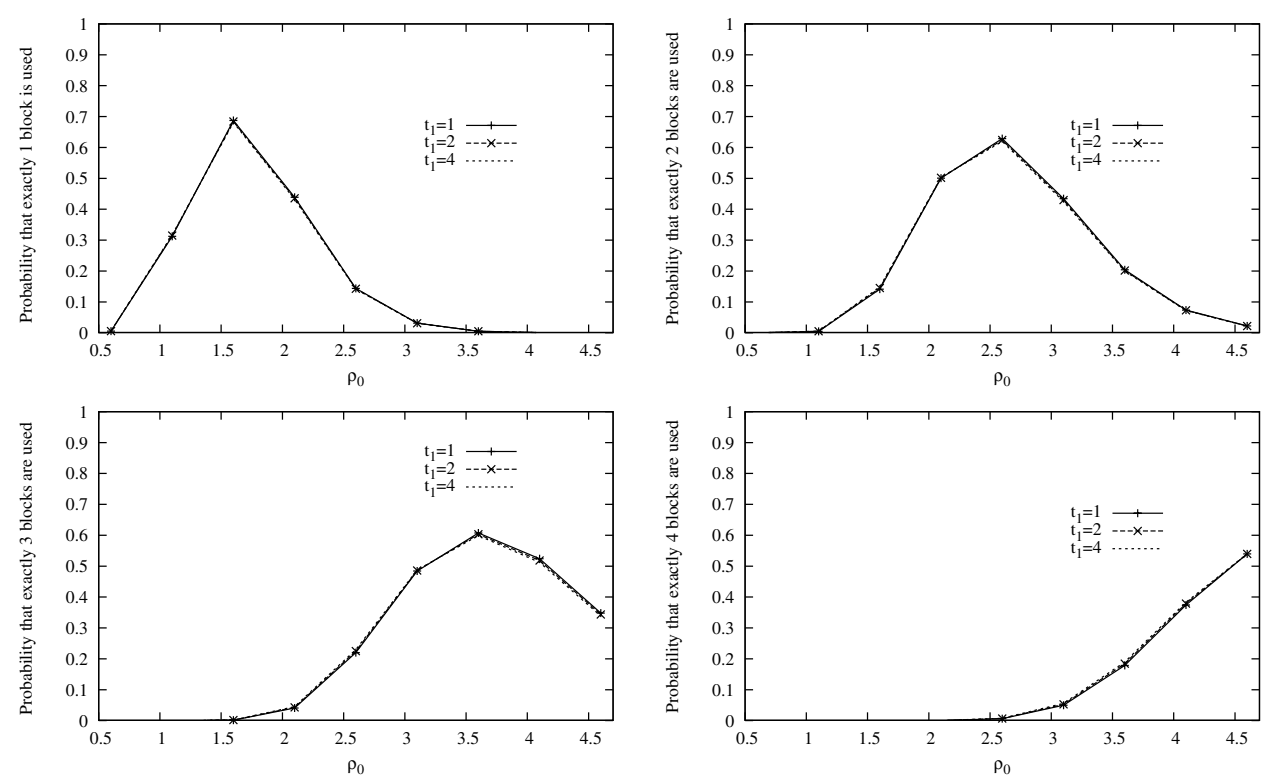

Fig. 4. Further performance measures for $t_{2}=6$.

of experience" is in the term of the blocking probability and the waiting time of subscribers. The observation is confirmed by the curves in Fig. 3. For example, at $\rho_{0}=1.1$, the blocking probability is approximately $0.013,0.007$, and 0.003 for $t_{1}=1,2$, and 4 , respectively. On the other hand, high values of $t_{1}$ result in a long renting time and a high renting fee an operator needs to pay for additional channel blocks. The same observation can be obtained concerning the impact of $t_{2}$ (see Fig. 5).

To consider the impact of a renting fee, the APR is computed as follows

$$
\mathrm{APR}=\alpha \times \bar{S}-\beta \times \bar{B},
$$

where $\alpha$ and $\beta$ are cost coefficients measured in cost units per time unit. Note that $\alpha \times \bar{S}$ can be interpreted as the revenue rate that the operator receives from the realization of calls, while $\beta \times \bar{B}$ is the renting fee due to the usage of rented frequency bands. Because there are $r$ channels in one frequency band, $r \times \alpha$ is the maximum revenue rate obtainable with the rent of one frequency band. It is reasonable to assume that the expected revenue from a rented channel should be higher than or equal to the cost of renting the channel. Furthermore, the operator should take into account the phenomenon that not all rented channels can be fully utilized. Therefore, $r \times \alpha \geq \beta$ holds, which is equivalent to $r \times \alpha=d \times \beta$ for $d \geq 1$. Note that $d=r \times \alpha / \beta$ is interpreted as the discount factor.

Figures 6-8 illustrate the impact of the discount factor $d$ on the APR. At low loads, the smaller the discount, the more it discourages the operator to rent additional channel blocks. At high loads, $d=1$ (no discount) still encourages the 


\section{2nd Reading}
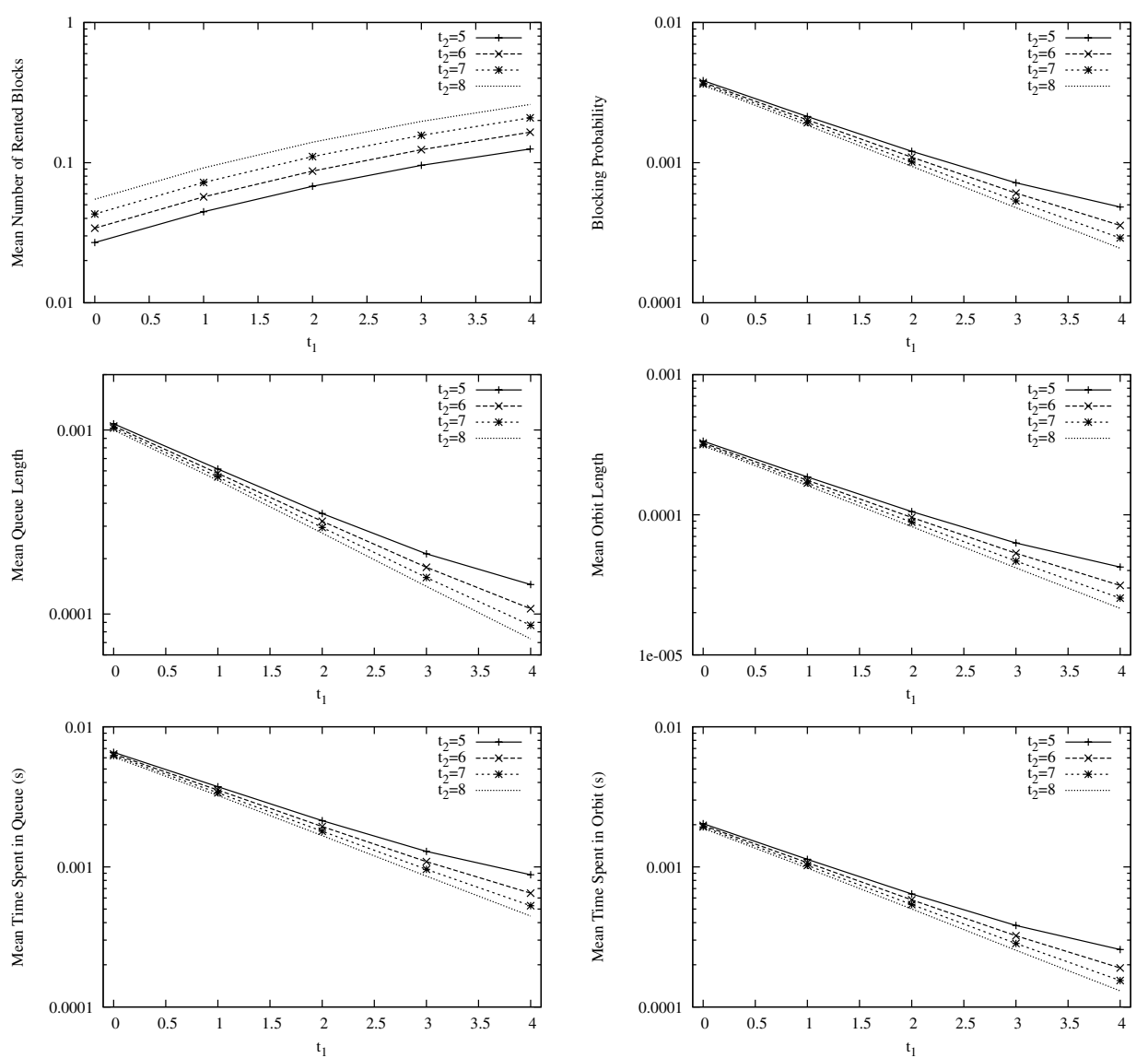

Fig. 5. Performance measures for $\rho_{0}=0.6$.

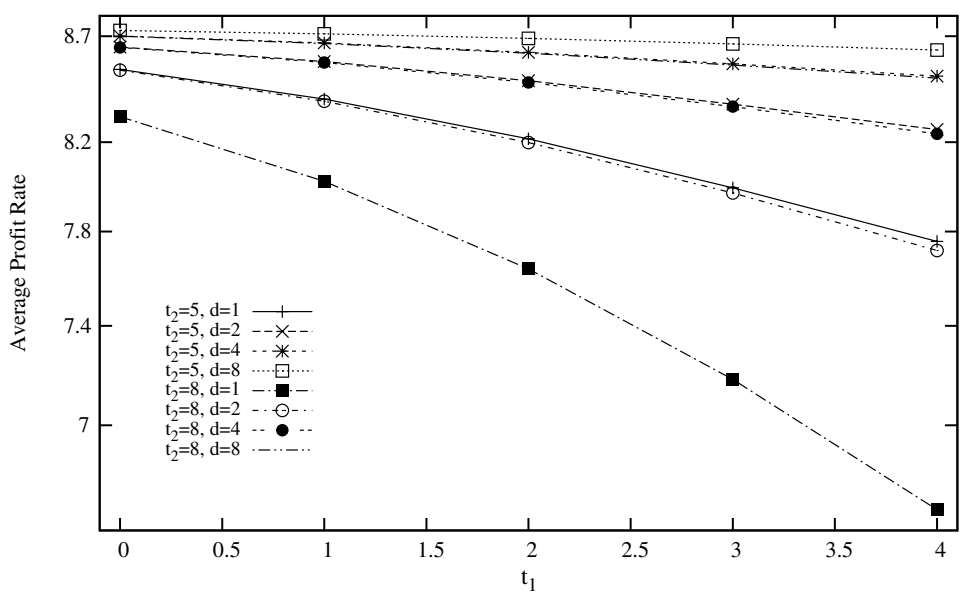

Fig. 6. APR versus $t_{1}$ and $d$ for $\rho_{0}=0.6, \alpha=1$ cost unit $/ \mathrm{s} \beta=r \times \alpha / d$. 


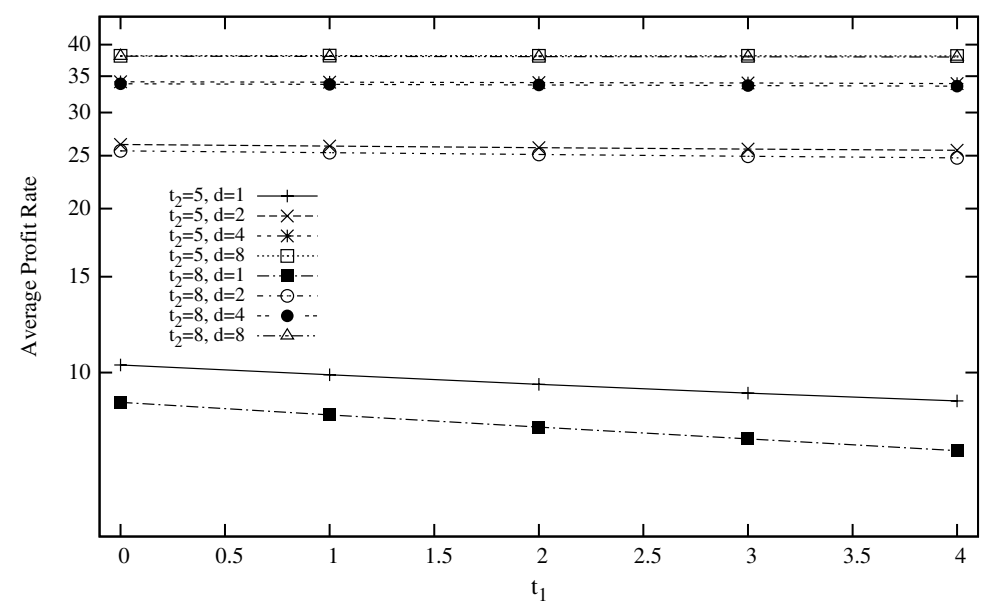

Fig. 7. APR versus $t_{1}$ and $d$ for $\rho_{0}=4.6, \alpha=1$ cost unit/s, $\beta=r \times \alpha / d$.

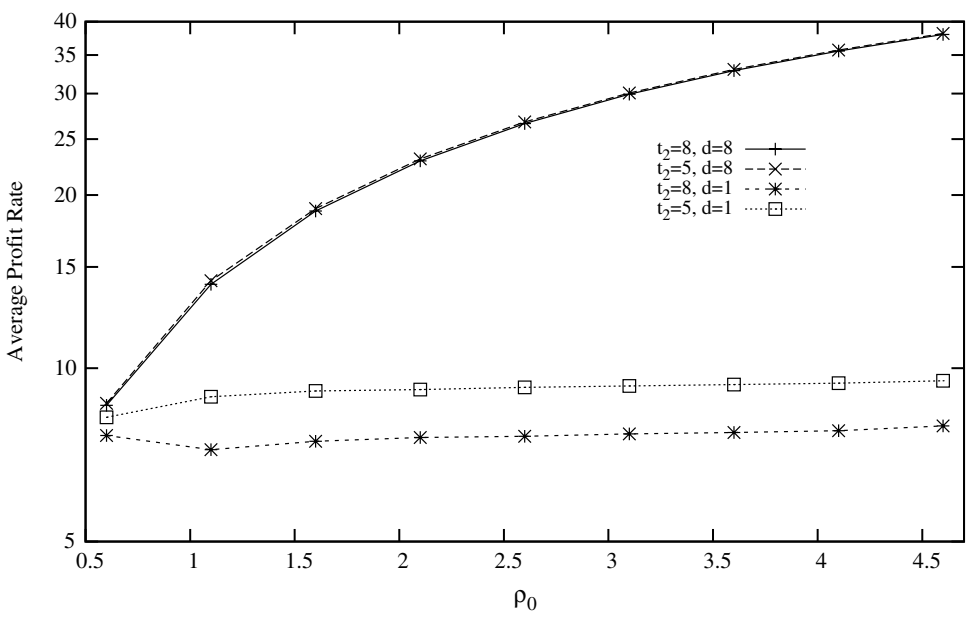

Fig. 8. APR versus $\rho_{0}$ and $d$ for $\alpha=1$ cost unit/s, $\beta=r \times \alpha / d$.

operator to make an early request for additional bands and release the rented bands as late as possible.

We plot the APR versus $\rho_{0}$ in Fig. 9 for three parameter sets: No retrials $\left(p_{o} \approx 0\right.$, $\left.p_{I o} \approx 0\right)$, modest retrials $\left(p_{o}=0.2, p_{I o}=0.4\right)$, and normal retrials $\left(p_{o}=0.4, p_{I o}=\right.$ $0.8)$. It is observed that the APR increases with a distinct retrial behavior because retrials increase the server utilization (see Fig. 10). However, retrials are annoying for the users, which may result in subscribers changing their service provider and consequently, in a long-term reduction of profit. This effect, nevertheless, is not yet included in our model. 


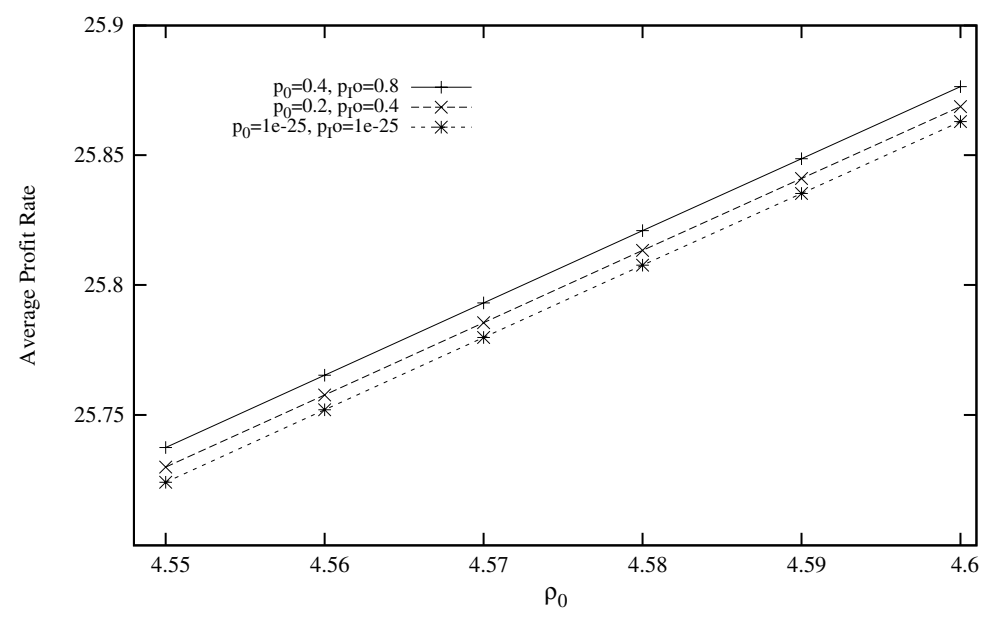

Fig. 9. Impact of retrials on APR for $\alpha=1$ cost unit/s, $t_{1}=2, t_{2}=5, d=2, \beta=r \times \alpha / d$.

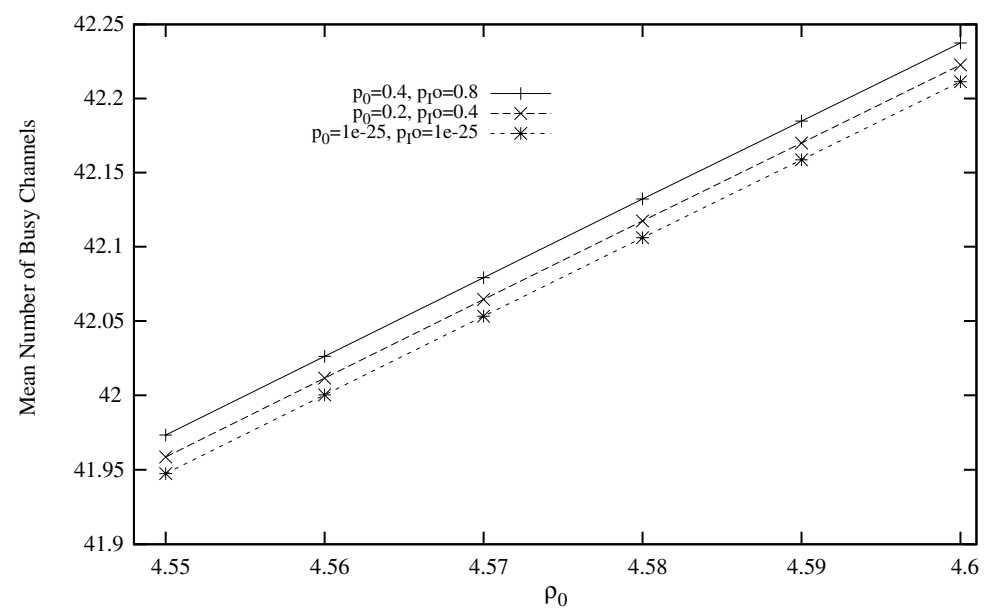

Fig. 10. Impact of retrials on the average number of busy channels for $\alpha=1$ cost unit $/ \mathrm{s}, t_{1}=2$, $t_{2}=5, d=2, \beta=r \times \alpha / d$.

\section{Conclusions}

We have proposed a finite-source retrial queueing model to evaluate the performance and the impact of spectrum renting in mobile cellular networks. We have investigated the impact of parameters on the performance of the system. The discount factor is introduced to take into account the renting fee. Numerical results show that it is still desirable to initiate a spectrum renting request even if there is no discount from the owners of frequency bands. 


\section{T. V. Do et al.}

To our best knowledge, this is the first proposal for the use of the theory of retrial queues to model spectrum renting. Many variants and generalizations of the presented model can be defined (e.g., the consideration of handover calls and guard channels, including non-exponential distributions), which will be investigated in our future works. We hope we can raise the attention of researchers to a sub-class of new retrial queueing models by shedding light on its application to the performance evaluation of mobile cellular networks.

\section{Acknowledgments}

The publication of T. V. Do was supported by the TÁMOP-4.2.2.C-11/1/KONV2012-0001 project. The project has been supported by the European Union, cofinanced by the European Social Fund.

The contributions by University of Passau are partially supported by the European Community's Seventh Framework Program through the SOCIONICAL project (FP7-ICT-2007-3,Grant No. 231288) and through the Network of Excellence EINS (FP7-ICT-2011-7, Grant No. 288021).

The work of T. Bérczes was supported by the European Union and the State of Hungary, co-financed by the European Social Fund in the framework of TÁMOP4.2.4.A/ 2-11/1-2012-0001 "National Excellence Program".

The research of J. Sztrik was supported by the European Union and the State of Hungary, co-financed by the European Social Fund in the framework of TÁMOP 4.2.4. A/2-11-1-2012-0001 "National Excellence Program".

\section{References}

Ajmone Marsan, M, GD Carolis, E Leonardi, R Lo Cigno and M Meo (2001). Efficient estimation of call blocking probabilities in cellular mobile telephony networks with customer retrials. IEEE Journal on Selected Areas in Communications, 19(2), 332-346.

Almási, B, J Roszik and J Sztrik (2005). Homogeneous finite-source retrial queues with server subject to breakdowns and repairs. Mathematical and Computer Modelling, 42(5-6), 673-682.

Artalejo, JR (1998). Retrial queues with a finite number of sources. Journal of Korean Mathematical Society, 35, 503-525.

Artalejo, JR (1999). Accessible bibliography on retrial queues. Mathematical and Computer Modelling, 30, 1-6.

Artalejo, JR (2010). Accessible bibliography on retrial queues: Progress in 2000-2009. Mathematical and Computer Modelling, 51(9-10), 1071-1081.

Artalejo, JR, A Economou and A Gómez-Corral (2007). Applications of maximum queue lengths to call center management. Computers and Operations Research, 34(4), 983-996.

Artalejo, JR and A Gómez-Corral (2008). Retrial Queueing Systems: A Computational Approach. Berlin, Heidelberg: Springer Verlag.

Artalejo, JR and MJ Lopez-Herrero (2010). Cellular mobile networks with repeated calls operating in random environment. Computers and Operations Research, 37(7), 1158-1166. 


\section{2nd Reading}

Artalejo, JR and MJ Lopez-Herrero (2012). The single server retrial queue with finite population: A BSDE approach. Operational Research, 12(2), 109-131.

Artalejo, JR, DS Orlovsky and AN Dudin (2005). Multi-server retrial model with variable number of active servers. Computers and Industrial Engineering, 48(2), 273-288.

Bolch, G, S Greiner, H de Meer and K Trivedi (2006). Queueing Networks and Markov Chains, 2nd Edition. New York: John Wiley \& Sons.

Buddhikot, MM (2007). Understanding dynamic spectrum access: Models, taxonomy, and challenges. In 2nd IEEE Int. Symp. New Frontiers in Dynamic Spectrum Access Networks, DySPAN 2007, pp. 649-663.

Ciardo, G, JK Muppala and KS Trivedi (1989). SPNP: Stochastic petri net package. In PNPM, pp. 142-151, IEEE Computer Society.

Do, TV (2010a). An efficient solution to a retrial queue for the performability evaluation of DHCP. Computers and Operations Research, 37(7), 1191-1198.

Do, TV (2010b). A new computational algorithm for retrial queues to cellular mobile systems with guard channels. Computers and Industrial Engineering, 59(4), $865-872$

Do, TV (2011). Solution for a retrial queueing problem in cellular networks with the fractional guard channel policy. Mathematical and Computer Modelling, 53(11-12), $2058-2065$.

Do, TV and R Chakka (2010). An efficient method to compute the rate matrix for retrial queues with large number of servers. Applied Mathematics Letters, 23, 638-643.

Do, TV, NH Do and R Chakka (2012). A new queueing model for spectrum renting in mobile cellular networks. Computer Communications, 35(10), 1165-1171.

Do, TV, NH Do and J Zhang (2013). An enhanced algorithm to solve multiserver retrial queueing systems with impatient customers. Computers and Industrial Engineering, 65(4), 719-728.

Economou, A and MJ Lopez-Herrero (2009). Mobile networks performance analysis of a cellular mobile network with retrials and guard channels using waiting and first passage time measures. European Transactions on Telecommunications, 20(4), 389-401.

Falin, G (1990). A survey of retrial queues. Queueing Systems, 7(2), 127-167.

Falin, G and J Templeton (1997). Retrial Queues. London: Chapman \& Hall.

Gandhi, S, C Buragohain, L Cao, H Zheng and S Suri (2008). Towards real-time dynamic spectrum auctions. Computer Networks, 52(4), 879-897.

German, R, C Kelling, A Zimmermann, G Hommel, TU Berlin and FPU Robotik (1995). TimeNET - a toolkit for evaluating non-Markovian stochastic petri nets. Performance Evaluation, 24, 69-87.

Gharbi, N and L Charabi (2012). Wireless networks with retrials and heterogeneous servers: Comparing random server and fastest free server discipline. International Journal on Advances in Networks and Services, 5(1-2), 102-115.

Gharbi, N and C Dutheillet (2011). An algorithmic approach for analysis of finite-source retrial systems with unreliable servers. Computers and Mathematics with Applications, 62(6), 2535-2546.

Greiner, S and G Bolch (1995). Modeling production lines with blocking, batch processing and unreliable machines using the Markov analyzer MOSES. In Proc. European Simulation Symp. ESS'95, pp. 303-309.

Hirel, C, B Tuffin and KS Trivedi (2000). SPNP: Stochastic Petri nets. version 6.0. In Proc. 11th Int. Conf. Computer Performance Evaluation: Modelling Techniques and Tools, pp. 354-357. London, UK: Springer-Verlag. 
Jabbari, B, R Pickholtz and M Norton (2010). Dynamic spectrum access and management dynamic spectrum management. IEEE Wireless Communications, 17(4), 6-15.

Kulkarni, VG and HM Liang (1997). Retrial queues revisited. Frontiers in Queueing: Models and Applications in Science and Engineering, pp. 19-34. Florida: CRC Press.

Lin, YB and I Chlamtac (2001). Wireless and Mobile Network Architectures. Wiley Computer Publishing, Indianapolis: John Wiley \& Sons, Inc.

Peha, JM (2009). Sharing spectrum through spectrum policy reform and cognitive radio. Proc. IEEE, 97, 708-719.

Perel, N and U Yechiali (2010). Queues with slow servers and impatient customers. European Journal of Operational Research, 201(1), 247-258.

Rappaport, TS (2002). Wireless Communications, Principles and Practice. Prentice-Hall.

Takacs, L (1962). Introduction to the Theory of Queues. New York: Oxford University Press.

Tran-Gia, P and M Mandjes (1997). Modeling of customer retrial phenomenon in cellular mobile networks. IEEE Journal on Selected Areas in Communications, 15(8), 14061414 .

Tzeng, S-S (2009). Call admission control policies in cellular wireless networks with spectrum renting. Computer Communications, 32(18), 1905-1913.

Tzeng, S-S and CW Huang (2010). Threshold based call admission control for QoS provisioning in cellular wireless networks with spectrum renting. In Novel Algorithms and Techniques in Telecommunications and Networking, Sobh, TM, KM Elleithy and A Mahmood (Eds.), pp. 17-22. Berlin Heidelberg: Springer.

Wang, J, L Zhao and F Zhang (2011). Analysis of the finite source retrial queues with server breakdowns and repairs. Journal of Industrial and Management Optimization, 7, 655-676.

Wüchner, P, J Sztrik and H de Meer (2009). Finite-source M/M/S retrial queue with search for balking and impatient customers from the orbit. Computer Networks, $53(8), 1264-1273$.

Yang, T and JGC Templeton (1987). A survey on retrial queues. Queueing Systems, 2, 201-233.

Zhang, F and J Wang (2013). Performance analysis of the retrial queues with finite number of sources and service interruptions. Journal of the Korean Statistical Society, 42(1), $117-131$

Zhang, F and J Wang (2012). Stochastic analysis of a finite source retrial queue with spares and orbit search. In $M M B / D F T$, Lecture Notes in Computer Science, JB Schmitt (Ed.), Vol. 7201, pp. 16-30. New York: Springer.

Tien Van Do is a Professor at Budapest University of Technology and Economics, and a leader of Communications Network Technology and Internetworking Laboratory. He obtained his habilitation at Budapest University of Technology and Economics, and the DSc title from the Hungarian Academy of Sciences. He has participated in a number of European research projects. He led various projects on network planning and software implementations that results are directly used for industry such ATM \& IP network planning software for Hungarian Telekom, GGSN tester for Nokia, performance testing program for the performance testing of the NOKIA IMS product, automatic software testing framework for Nokia Siemens Networks. His research interests are queuing theory, telecommunication networks, cloud computing, performance evaluation, and planning of ICT Systems. 
Patrick Wüchner is a project assistant, research associate, and a teaching assistant at the University of Passau, Germany in the research group of Computer Networks and Communications, chaired by Professor Hermann de Meer. He received MSc degree in Computer Science (Diplom-Informatiker) in 2004 from the University of Erlangen-Nuremberg, Germany. Currently, he is pursuing his PhD. His main research interest is mathematical performance and reliability modeling of computer systems, communication networks, and self-organizing systems.

Tamás Bérczes received his MSc degree in Mathematics in 2000 at the University of Debrecen, Hungary. He received his PhD in 2011. He is currently working as an assistant professor at the Department of Informatics Systems and Networks of the same university. His primary research interests are performance analysis of retrial queues and their applications.

János Sztrik is a Professor at the Faculty of Informatics, University of Debrecen, Hungary. He obtained the Candidate of Mathematical Sciences Degree in Probability Theory and Mathematical Statistics in 1989 from Kiev State University, Kiev, USSR; Habilitation from the University of Debrecen in 1999; doctoral degree from the Hungarian Academy of Sciences, Budapest, 2002. His research interests are in the field of production systems modeling and analysis, queueing theory, reliability theory, and computer science.

Hermann de Meer has been appointed as a Professor of Computer Science (director of Computer Networks and Communications Lab) at the University of Passau in sub-sequence of his Readership at the University College London (UCL), UK, in Networks and Distributed Systems. Before that, he was a visiting Professor at the Columbia University, NYC, USA, Scholar of the Deutsche Forschungsgemeinschaft (DFG) and Postdoc at the University of Texas at Austin, TX, and Duke University, NC, USA. He had received his PhD from the University of Erlangen-Nuremberg and his habilitation from Hamburg University, Germany. He is co-authoring a textbook on Queueing Networks and Markov Chains, John Wiley and was chairing and organizing editions of workshops and conferences like IWSOS, IWQoS, and ACM e-Energy. He has been awarded various national and international research grants, the focus of which have been on Performance Modeling, Energy Efficiency, Smart Grid, and IT Security. 